\title{
Context-Aware Multicast Protocol for Emergency Message Dissemination in Vehicular Networks
}

\author{
Alvin Sebastian, Maolin Tang, Yanming Feng, and Mark Looi \\ School of Electrical Engineering and Computer Science, Queensland University of Technology, GPO Box 2434, 2 George Street, \\ Brisbane, QLD 4000, Australia \\ Correspondence should be addressed to Alvin Sebastian, a.sebastian@qut.edu.au
}

Received 17 January 2012; Revised 21 March 2012; Accepted 19 June 2012

Academic Editor: T. A. Gulliver

Copyright ( $(2012$ Alvin Sebastian et al. This is an open access article distributed under the Creative Commons Attribution License, which permits unrestricted use, distribution, and reproduction in any medium, provided the original work is properly cited.

Road traffic accidents can be reduced by providing early warning to drivers through wireless ad hoc networks. When a vehicle detects an event that may lead to an imminent accident, the vehicle disseminates emergency messages to alert other vehicles that may be endangered by the accident. In many existing broadcast-based dissemination schemes, emergency messages may be sent to a large number of vehicles in the area and can be propagated to only one direction. This paper presents a more efficient contextaware multicast protocol that disseminates messages only to endangered vehicles that may be affected by the emergency event. The endangered vehicles can be identified by calculating the interaction among vehicles based on their motion properties. To ensure fast delivery, the dissemination follows a routing path obtained by computing a minimum delay tree. The multicast protocol uses a generalized approach that can support any arbitrary road topology. The performance of the multicast protocol is compared with existing broadcast protocols by simulating chain collision accidents on a typical highway. Simulation results show that the multicast protocol outperforms the other protocols in terms of reliability, efficiency, and latency.

\section{Introduction}

Vehicular ad hoc networks (VANETs) are wireless ad hoc networks operating in a vehicular environment that involves communication between vehicle-to-vehicle (V2V) and vehicle-to-roadside infrastructure (V2I). The deployment of VANET is feasible in the near future due to rapid advances in wireless communication technologies, particularly the IEEE 802.11 wireless LAN. The most potential technology that can provide robust and reliable $\mathrm{V} 2 \mathrm{~V}$ and V2I communication is most likely will be based on the IEEE 802.11p standard [1] and the IEEE 1609 Family of Standards for Wireless Access in Vehicular Environments (WAVE) [2]. VANETs enable the development of vehicular safety applications that can improve road safety significantly. The most prominent safety application that aims to reduce the number of traffic accidents is a cooperative collision warning system (CCWS). A CCWS works by having vehicles exchange safety messages using $\mathrm{V} 2 \mathrm{~V}$ communication to detect any possible collision and warn the drivers accordingly. Several concepts and prototypes of CCWSs have been proposed and developed [3-6], demonstrating the technical feasibility of CCWS.

Several performance evaluations of the IEEE 802.11p standard indicate that the standard is able to achieve promising latency and reliability compared to safety application requirements [7-10]. However, the standard alone can not ensure time-critical message dissemination in dense road traffic conditions, such as in traffic jams. Dense traffic conditions imply a high communication channel load that can significantly deteriorate the communication performance. Therefore, it is necessary to develop application level protocols that use the communication channel more efficiently to ensure fast and reliable safety message dissemination in any traffic conditions.

In designing the application level protocols for safety purposes, there are two fundamental types of messages that must be considered: Routine Safety Messages (RSMs) and Event Safety Messages (ESMs) [11]. RSMs, also known as beacons, are status update messages regularly sent by vehicles, 
so that every vehicle can realize the state of neighbor vehicles and warn the driver of any possible collision. ESMs, or emergency warning messages, are notification messages sent by a vehicle to warn other vehicles when certain endangering events occur. To improve the overall communication performance, it is important to have efficient communication protocols for both types of messages. However, this work particularly focuses on the problem of disseminating ESMs in a reliable, efficient, and timely manner.

ESMs are especially useful to mitigate a multivehicle chain collision because they can propagate faster than visual indicators such as tail brake lights [12]. An example of safety applications designed to prevent such accidents by using ESMs is an Electronic Emergency Brake Light (EEBL) application $[10,13]$. An EEBL application will trigger ESM dissemination if it detects a deceleration that exceeds a certain threshold. A vehicle that decelerates rapidly may endanger its following vehicles and therefore must send ESMs to other endangered vehicles in a timely manner with the highest priority. Upon receiving an ESM, any endangered vehicle informs its driver of the possible dangerous situation through on-board interfaces (audio, visual, etc.). Being made aware of the dangerous situation, the driver can perform an evasive action accordingly.

Depending on the road traffic conditions, ESMs may need to be disseminated along a roadway beyond the coverage area of the original sender. There are many cases where a multivehicle collision involves a lot of vehicles. As an example, an accident in Germany in 2009 involved more than 200 vehicles on a $30 \mathrm{~km}$ stretch of road [14]. In such cases, the distance required to reach all the relevant vehicles far exceeds the reliable communication range of an IEEE $802.11 \mathrm{p}$ compliant radio, which is only $505 \mathrm{~m}$ at the maximum [15]. Therefore, multihop ESM dissemination is necessary in some situations to anticipate any possible collision.

Existing multihop ESM dissemination protocols generally use a scoped-broadcast technique in which ESM dissemination is limited to a specific area and direction. They are not efficient as they may still send ESMs to vehicles that are not endangered and they cannot prioritize the receivers based on their critical time to avoid collision. In addition, they are designed to operate only in a specific road traffic situation, which is a straight road segment such as a highway, where only two directions are considered: forward or backward. This work presents a more efficient dissemination protocol that can disseminate ESMs to any directions as necessary. The protocol operates in the application layer, which makes it possible to utilize the contextual knowledge such as the state of neighboring vehicles [11].

The original contribution of this work is a new contextaware multicast routing strategy that can disseminate ESMs efficiently. The objective is to reduce unnecessary transmissions and to deliver ESMs to endangered vehicles as fast as possible to prevent vehicle collisions. The multicast routing strategy combines a tree-based routing method with a contention-based method. The strategy uses context information processed from the states of neighboring vehicles that are obtained via RSMs. Using the context information, a multicast tree is generated by finding the most efficient routes from a sender node to all the receiver nodes. The problem of finding the multicast tree is formulated as a minimum delay tree problem, which can be solved using Dijkstra's shortest path algorithm [16]. To the best of our knowledge, this multicast concept for an ESM protocol has never been proposed previously in the literature. The performance of the multicast protocol is evaluated by simulation and compared with existing broadcast protocols. The simulation results show that using the multicast protocol resulted in fewer vehicles collisions in some scenarios compared to the other protocols.

The rest of this paper is organized as follows. Section 2 presents an overview of relevant protocols that address the problem of multihop ESM dissemination. Section 3 introduces a multicast approach in optimizing the multihop ESM dissemination and proposes a new context-aware multicast protocol that can efficiently disseminate ESMs. Section 4 describes the performance evaluation of the proposed protocol and Section 5 analyzes the simulation result and the advantages of the proposed multicast protocol. Finally, Section 6 concludes this work and proposes future research direction.

\section{Related Work}

The ESM dissemination problem is related to routing problems in wireless networks. In general computer networks, there are three basic types of routing schemes: unicast, broadcast, and multicast. Unicast routing is used to deliver a message to a specified node. Broadcast routing is used to deliver a message to all nodes in the network. Multicast routing is used to deliver a message to a group of nodes that have expressed interest in receiving the message. In wireless networks with omnidirectional antennas, one-hop communication is inherently a broadcast at the physical level, and therefore using unicast, broadcast, or multicast routing for one-hop communication consumes the same network resources. However, for multihop communication, multicast routing is more efficient than plain broadcast routing.

In VANET, existing routing schemes can be broadly categorized into general purpose and safety purpose routing. General purpose routing is used to disseminate any type of data such as traffic information or multimedia streams. In principle, it requires finding and establishing a route from a sender to a receiver, and using the established route to deliver the data, which may contain many messages. There are two basic approaches to finding the route: (1) a reactive approach finds the route on-demand by broadcasting a hello message to all communication nodes (e.g., vehicles) at the time a message needs to be sent; (2) a proactive approach maintains up-to-date network information and uses the information to find the route when needed.

Safety purpose routing is used to disseminate data important for road safety, such as ESMs. Safety purpose routing requires lower latency and higher reliability compared to general purpose routing. Existing safety purpose routing schemes use a similar approach to the reactive approach with 
one significant difference. Given the size of a safety message is relatively small, there is no need to find and establish the route before sending the actual message. Instead, they use a connection-less approach that broadcasts a safety message as a hello message itself. A comprehensive comparison of existing ad hoc routing protocols in vehicular environment shows that the connection-less approach is suitable for safety applications because its relatively low latency [17].

The simplest method to disseminate an ESM in a multihop manner is by using a plain broadcast scheme such as flooding. In a flooding scheme, messages are disseminated to all nodes by having all the nodes retransmit or relay received messages. A flooding scheme is highly inefficient and may cause a broadcast storm problem [18]. A broadcast storm can overload the limited channel capacity, causing channel congestion that reduces communication reliability. Therefore, various scoped broadcast schemes that can improve the efficiency of multihop safety communication have been proposed in the literature. The scoped broadcast schemes use various techniques to limit the number of transmissions, reduce unnecessary transmissions, and improve reliability.

Yang et al. [19] introduced the concept of an abnormal vehicle and the state transition mechanism to disseminate ESMs. A vehicle becomes an abnormal vehicle when an emergency event is happening to the vehicle itself or when the vehicle is reacting to other abnormal vehicles nearby. As long as a vehicle is marked as an abnormal vehicle, it repeatedly broadcasts the ESMs to increase the successful reception rate. Once an abnormal vehicle resumes its regular movement, the vehicle is no longer an abnormal vehicle and it returns back to its normal state. The dissemination method is not designed to propagate ESMs to all relevant vehicles as fast as possible. In addition, the broadcast repetition may result in unnecessary transmissions.

There are several techniques commonly used in the literature to improve the efficiency of ESM dissemination. Most of them are based on an approach in which a vehicle that receives an ESM decides whether to forward, ignore, or use the received ESM. To reduce the number of retransmissions, the number of hops can be minimized by selecting the optimal forwarder. Implicit acknowledgment is another technique to limit the number of repetitions while ensuring reliability. The technique introduces a contention duration before rebroadcasting the same message. When a vehicle detects that the same message has been rebroadcast by another vehicle, it will abort the rebroadcast attempt.

The methods used to select a relay and calculate the contention duration can be based on distance only [2022], distance with a dedicated back-off procedure [27, 29], distance with a random contention window [23], distance with a probability factor [24], directions with a random duration [12], a combination of distance and directions [25], or a predefined area [26]. One of the position-based methods also considers the angle between vehicles [27]. Most of the ESM dissemination protocols in the literature do not consider the use of RSMs. They assume that the information about the receivers and their location is not available. In such a case, the scoped broadcast strategies are the most suitable option. Torrent-Moreno et al. [28] proposed an ESM protocol that makes use of information obtained from the RSM. The protocol works by nominating a specific forwarder for every transmission to minimize the delay caused by the contention duration.

One of the key techniques used by existing schemes is to limit the ESM dissemination into a specific area or direction. The intended receivers (i.e., the endangered vehicles) are assumed to be all the vehicles that are inside a region behind the sender $[12,19,22,23,28,29]$, behind the sender but moving in the same direction [27], within a specified message direction [25], or inside a predefined risk zone $[20,26]$. All the aforementioned schemes are designed for a highway or straight road scenario and only consider two directions: forwards or backwards. Since interactions among vehicles are not considered, ESMs can be disseminated to irrelevant vehicles and vehicles cannot be prioritized based on their critical time to avoid a collision. Taha and Hasan [30] proposed a scheme that gives more priority to a vehicle in the most danger to become a relay node. The scheme does not reduce redundant transmissions to improve the dissemination efficiency. Priority is determined based on a time headway model, which is one of the car following models, implying that such a scheme can operate only in highways.

In contrast to the prior works, this study is based on the idea of a multicast scheme in which an abnormal vehicle should send ESMs only to vehicles that are possibly endangered by the abnormal vehicle's maneuver. This paper extends our previous work [31] with a different routing strategy, a new protocol description, and a complete performance evaluation that includes direct comparison with existing ESM protocols. The previous work addressed the problem of multicast routing with a dynamic transmission range while this work further investigates the special case of the problem in which the transmission range is static instead of dynamic.

\section{Context-Aware Multicast Approach}

3.1. Multihop ESM Dissemination. A vehicle that encounters an emergency situation becomes an abnormal vehicle. To reduce unnecessary transmissions, an abnormal vehicle should send the ESMs only to relevant vehicles instead of all vehicles. The relevant vehicles are vehicles that are endangered by the abnormal vehicle's maneuver. In wireless communication, this strategy is useful only for multihop transmissions because a single-hop transmission using is basically a broadcast to all vehicles within the radio transmission range. However, to prevent multivehicle chain collisions, multihop transmissions are often needed to propagate ESMs along a roadway longer than a single vehicle's transmission range.

From the perspective of communication, the abnormal vehicle is the sender node and the relevant vehicles are the receiver nodes, which can be considered as a multicast group. For example, in a scenario shown in Figure 1, assume that vehicle $a$ performs a sudden movement at time $t$ that endangers vehicles $b, c$, and $g$. Therefore, vehicle $a$ sends 


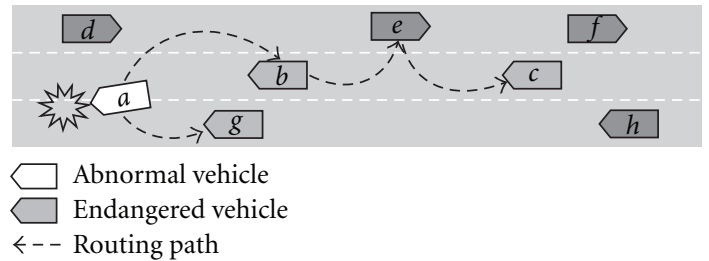

FIgURE 1: Illustration of multihop routing for ESMs.

ESMs to vehicles $b, c$, and $g$ at time $t$. Vehicle $a$ does not need to send the ESMs to vehicles $d, f$, and $h$ because they are not in immediate danger. Although vehicle $h$ is in the same lane as vehicle $g$, vehicle $h$ may not be endangered if its speed is slower than vehicle $g$ and the distance between them is large enough. A vehicle that is not in danger can receive and relay the ESMs. In this example, vehicle $e$ is the relay node to forward the ESMs to vehicle $c$.

This study assumes that a driver may or may not overreact to the alert given by a CCWS. Therefore, a vehicle that receives an ESM may react by braking abruptly (very high deceleration) or by braking sensibly (convenience deceleration) to achieve the safe distance from the leading vehicle. In a case when a receiving vehicle brakes abruptly, it will become another abnormal vehicle. To ensure safety, the new abnormal vehicle will send a new ESM to a new upto-date set of endangered vehicles. In the previous example, vehicle $g$ receives an ESM from vehicle $a$ at time $t$. Vehicle $g$ will start braking at time $t^{\prime}=t+t_{r}$, where $t_{r}$ is the driver's reaction time. If vehicle $g$ brakes abruptly, it becomes a new abnormal vehicle that triggers a new ESM dissemination. If, for example, vehicle $h$ becomes endangered by the action of vehicle $g$ at time $t^{\prime}$, vehicle $g$ will send a new ESM to vehicle $h$. If there are no endangered vehicles, vehicle $g$ does not need to send a new ESM.

An ESM is only useful if it is received within a certain time that allows a vehicle to brake and stop before crashing with its leading vehicle. In order to reduce the number of vehicle collisions, the relay node must be selected in such a way so that the end-to-end delay from the sender node to every receiver node is minimized. In a tree-based multicast routing, a sender computes a multicast tree that represents routing paths from the sender to every receiver. This study proposes the use of a minimum delay multicast tree to provide routing paths with the least end-to-end delay for ESM dissemination.

3.2. System Assumptions. The multicast approach is designed for a typical CCWS in which a vehicle can determine its own position on the road using a combination of a Differential Global Positioning System (DGPS) and internal motion sensors. Prior studies with field tests have indicated that current positioning technology can provide centimeter-level accuracy under good conditions $[4,5]$. Other technologies such as a relative distributed ad hoc localization can provide relative positioning by measuring the signal strength of wireless communication devices [32, 33]. By using data fusion techniques $[34,35]$ that combine various localization techniques, it is reasonable to assume that a vehicle can obtain position coordinates relative to other vehicles with an accuracy suitable for safety purposes.

In this work, we assume that every vehicle is equipped with a wireless communication device that implements the IEEE 802.11p/WAVE standards, which defines the protocols for PHY, MAC, and network layers. The radio transmission range provided by the device is determined by the transmission power. For practical purposes, our simulations assume a constant transmission power is used by all vehicles. However, the multicast approach is designed by considering that a vehicle may have a different transmission range than others. We also assume that the communication devices operate in ad hoc mode and there is no roadside infrastructure available.

3.3. Problem Definitions. The communication network is modeled as a weighted directed graph $G=(V, E)$, where $V$ is a set of communication nodes representing the vehicles and $E$ is a set of directed edges representing the communication links between the nodes. A directed edge $e=\langle u, v\rangle \in E$ if and only if node $v$ can receive packets from node $u$, where $u, v \in$ $V$ and $u \neq v$. A nonnegative real-valued function is associated with each node $v \in V: \delta(v): V \rightarrow \mathbb{R}^{+}$, which represents the estimated one-hop delay for every packet relayed through node $v$. Let $s \in V$ be the initial sender of an ESM, and let $R \subseteq V-\{s\}$ be the set of receivers. Nodes belonging to $V \backslash(R \cup\{s\})$ may become relay nodes, that is, they are involved in forwarding the ESM, or they may remain isolated without receiving or transmitting any signal. A multicast tree $T(s, R)=\left(V_{T}, E_{T}\right)$, where $V_{T} \subseteq V$ and $E_{T} \subseteq E$, is a tree rooted at $s$ connecting all receiver nodes in $R$.

Let $P_{T}(s, r)$ be a unique path in the tree $T$ from the sender node $s$ to a receiver node $r \in R$. The set of nodes on the path $P_{T}(s, r)$ is defined as $U\left(P_{T}(s, r)\right)$. The total end-to-end delay from sender node $s$ to node $r \in R$ is defined as the sum of the delay of nodes in $U\left(P_{T}(s, r)\right) \backslash\{r\}$, that is,

$$
\delta\left(P_{T}(s, r)\right)=\sum_{v \in\left(U\left(P_{T}(s, r)\right) \backslash\{r\}\right)} \delta(v) .
$$

The maximum total end-to-end delay of the tree $\delta\left(P_{T}(s, R)\right)$ is defined as

$$
\delta\left(P_{T}(s, R)\right)=\max _{r \in R} \delta\left(P_{T}(s, r)\right) .
$$

Based on the previous definition, the minimum delay multicast tree $T(s, R)$ can be defined as a tree that has

$$
\min \delta\left(P_{T}(s, R)\right)
$$

3.4. Context Information. The minimum delay tree problem requires several input parameters and functions that depend directly or indirectly on the context information. Context information is defined as the road traffic and communication network situation perceived by the abnormal vehicle at the time an ESM needs to be sent. In particular, the context information includes the network topology, end-to-end communication delay, sender node, and receiver nodes. Each 
vehicle maintains its own context information which may or may not differ to other vehicles.

A vehicle generates or updates the context information by processing the tracked state information of the neighboring vehicles. The state information is obtained by listening to the RSMs (beacons) periodically broadcast by other vehicles. An RSM contains a timestamp and a unique identifier (such as a MAC address), the most current motion properties (position, speed, heading, and deceleration capability), and the transmission power of the vehicle. Each time a vehicle $v$ receives an RSM from vehicle $u$, vehicle $v$ updates its knowledge about vehicle $u$, which includes the network topology, delay history, and other context information.

Depending on the road traffic density and conditions, knowledge about up to $n$-hop neighboring vehicles may be needed. The number of hops $n$ can be a predetermined constant or a variable dynamically set at real time. There are several existing techniques $[22,36]$ that can be used to obtain the knowledge of more than one-hop neighbors. To avoid flooding, the $n$-hop information is aggregated and piggybacked into an extended RSM [28, 37]. This study assumes the use of an existing multihop beaconing scheme [36] to provide up to 4-hop neighbor information, which approximately corresponds to a coverage of two kilometers in each direction on a highway. The scheme is able to efficiently disseminate RSMs over multiple hops by suppressing redundant retransmissions, multiplexing messages, and scheduling transmission times dynamically.

3.4.1. Network Modeling. In a wireless networking environment, a receiver can successfully receive a message if the receiver is located within the sender's coverage area. For simplicity, the coverage area is modeled as a planar circle where its radius is the transmission range of the sender, as illustrated in Figure 2. The state of the network connections, represented by the network graph $G$, is estimated using the position and the maximum transmission range of each node. The maximum transmission range is the estimated communication range that can be achieved using the maximum transmission power.

Let $d_{u v}=d_{v u}$ be the distance between node $u \in V$ and node $v \in V$, and $R_{u}^{t x}$ and $R_{v}^{t x}$ be the maximum transmission range of node $u$ and $v$, respectively. The distance between node $u$ and node $v$ with Cartesian coordinates $\left(x_{u}, y_{u}\right)$ and $\left(x_{v}, y_{v}\right)$, respectively, is

$$
d_{u v}=\sqrt{\left(x_{u}-x_{v}\right)^{2}+\left(y_{u}-y_{v}\right)^{2}} .
$$

There exists a communication link represented by directed edge $\langle u, v\rangle \in E$ between node $u$ and node $v$ if $d_{u v} \leq R_{u}^{t x}$, which means that node $v$ can receive a message from node $u$. Given the set of nodes $V$, the set of edges $E$ can be generated by enumerating all the pairs of nodes in $V$. Algorithm 1 describes the procedure to generate the network graph.

3.4.2. Estimating Communication Delay. The total end-toend delay $\delta\left(P_{T}(s, r)\right)$ defined in (1) is the sum of the delay at each node between the sender (inclusive) and the receiver (exclusive). It depends on the number of intermediary

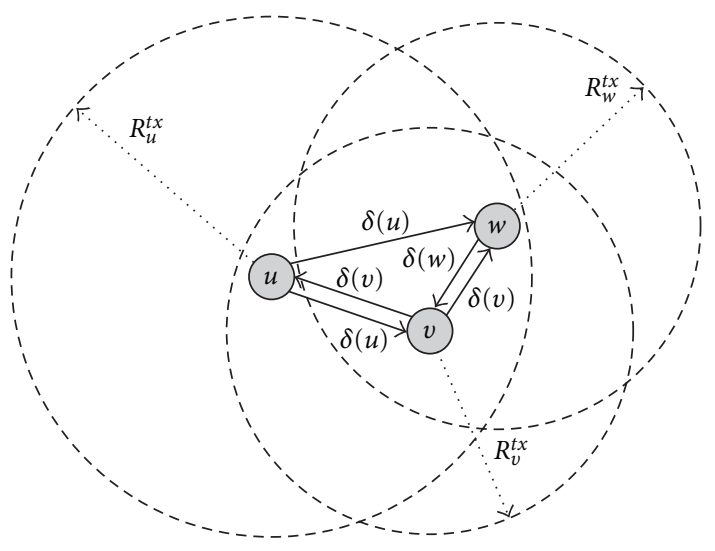

FIGURE 2: Illustration of the network modeling.

nodes between them and the delay experienced at each intermediary node (one-hop delay). To measure the onehop delay, we use an estimation method adopted from existing work in MANET QoS routing [38]. The one-hop delay $\delta$ is estimated by measuring the value of actual delays experienced by the RSMs. All of the nodes are assumed to have synchronized clocks via the GPS. Each time an RSM is created, it is timestamped with the current creation time. When a node $u$ receives an RSM from any other node $v$, node $u$ calculates the one-hop delay $\delta(v)$ by subtracting the value of the timestamp from the received time. The measured one-hop delay mostly consists of the MAC queuing and transmission delay caused by the MAC protocol back-off mechanism. Note that the priority of RSMs is assumed to be lower than the priority of ESMs. Therefore, the actual delay experienced by an ESM is expected to be less than the estimated delay.

3.4.3. Identifying the Sender Node. The sender node is an abnormal vehicle that initiates the ESM dissemination. There are two cases where a vehicle needs to send an ESM.

(1) A sudden change of vehicle state or unexpected circumstances experienced by the vehicle. A sudden change of vehicle state can be defined as a change in motion that exceeds a certain threshold, such as rapid deceleration or sudden change of direction. Unexpected circumstances are other dangerous factors that may cause an accident, such as engine breakdown, braking failure, or any other vehicle malfunction.

(2) An inevitable collision with any other vehicle based on the current state of vehicles. It is possible that a collision may happen without any sudden maneuver. For example, in a car following scenario, if the leading vehicle moves slower than the following vehicle, they will eventually collide. This kind of accident is most likely to be caused by inattentive drivers.

In a case when a collision involves two or more vehicles, multiple abnormal vehicles may initiate the same ESM dissemination at the same time. To reduce redundant transmission, any abnormal vehicle must wait for a very 


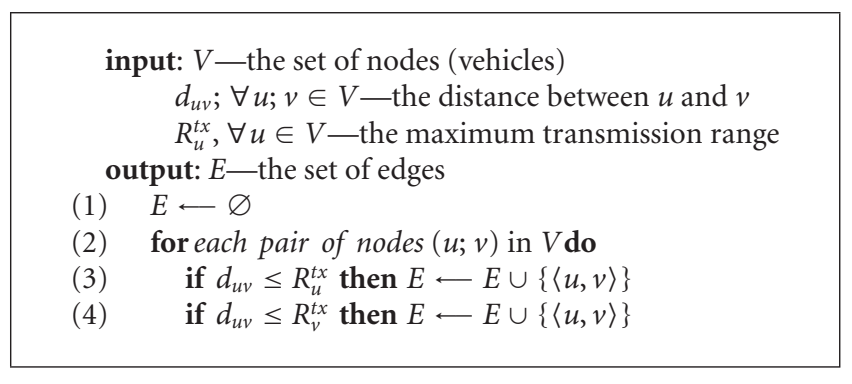

Algorithm 1: Generating the network graph.

short random time before sending a new ESM. If another ESM that describes the same event is received from another vehicle within the waiting period, the new ESM is discarded and will not be sent.

3.4.4. Identifying Receiver Nodes. The receiver nodes are vehicles that will be endangered by the course of an abnormal vehicle or the sender node. To identify the receiver nodes, a sender needs to know the relevant or endangered vehicles based on the current road traffic situation. To determine the endangered vehicles, an abnormal vehicle generates or updates a vehicle interaction graph [39] at the time of the emergency event. The interaction graph represents the interaction between multiple vehicles in a specific region and at a point in time. It provides context information on how vehicles interact with each other.

The interactions among multiple vehicles are modeled as a directed graph $\mathcal{G}=(\mathcal{V}, \mathcal{E})$, where $\mathcal{V}$ is a set of nodes representing vehicles and $\mathcal{E} \subseteq\left\{\left\langle v_{i}, v_{j}\right\rangle: v_{i}, v_{j} \in\right.$ $\left.\mathcal{V}, v_{i} \neq v_{j}\right\}, 1 \leq i, j \leq n$ is the set of directed edges representing interactions between vehicles. A directed edge $\left\langle v_{i}, v_{j}\right\rangle \in \mathcal{E}$ represents an interaction between $v_{i}$ and $v_{j}$, where $v_{i}$ influences or endangers $v_{j}$. Each vehicle maintains its own interaction graph $g$ by defining the set of vertices $\mathcal{V}$, which represents the neighboring vehicles, and the set of edges $\mathcal{E}$, which represents the interactions.

In an ad hoc environment, each vehicle tracks the motion state of its neighbor vehicles via the exchange of RSMs. Given the set of neighboring vehicles $\mathcal{V}$ tracked by each vehicle, an interaction graph $g$ is constructed by generating the set of edges $\mathscr{E}$. Initially, each vehicle $v$ creates a graph $\mathscr{g}=(\mathcal{V}, \mathscr{E})$, where $\mathcal{V}=\{v\}$ and $\mathcal{E}=\varnothing$. Every time a vehicle $v$ receives an RSM from other vehicle $v_{i} \in \mathcal{V}^{\prime}$, vehicle $v$ updates its interaction graph $g$ by enumerating each vehicle $v_{j} \in \mathcal{V}$, and calculating the interactions between $v_{i}$ and $v_{j}$ for each pair $\left(v_{i}, v_{j}\right), i \neq j$ based on the vehicle state information. Depending on the calculation result, an edge $\left\langle v_{i}, v_{j}\right\rangle,\left\langle v_{j}, v_{i}\right\rangle$, or both edges may be added to the set of edges $\mathscr{E}$.

A vehicle interacts with other vehicle if there is a possibility of collision between them. A possible collision is determined by calculating a route contention between a pair of vehicles and an avoidance time based on their motion state such as position coordinates, speed, acceleration, deceleration, heading, and trajectory. The general principle is that there is no interaction defined as long as the driver has more than enough time to evade a collision.

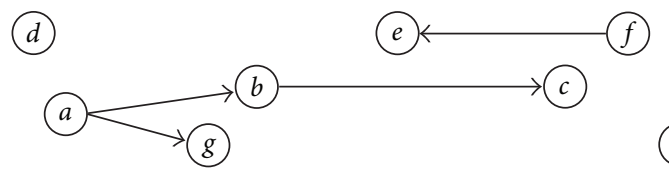

(h)

Figure 3: Example of an interaction graph given a traffic situation as shown in Figure 1.

There are three distinct cases that need to be considered in order to determine whether there is any interaction between any pair of vehicles. The first case is following, where both vehicles are traveling in the same direction. The second case is opposite, where both vehicles are traveling in the opposite direction. The third case is intersects, for any other conditions besides those previous two cases. The technical details on how to calculate the interaction between vehicles can be found in the paper [39].

The interaction graph is responsible to model any possible road traffic scenarios, allowing us to design a generalized communication protocol. Given the interaction graph $g$ and the sender node $s$, a set of receiver nodes $R$ can be obtained by performing a breadth-first search (BFS) algorithm from $s$. As an example, Figure 3 shows a possible interaction graph that represents the traffic situation shown in Figure 1. An arrow from node $a$ to node $b$ means that $b$ is endangered by $a$. Given the example interaction graph and node $a$ as the sender (abnormal vehicle), the receivers (endangered vehicles) are identified as nodes $b, c$, and $g$ by following the arrows from node $a$ in the interaction graph.

3.5. Context-Aware Multicast Protocol. A Context-aware multicast routing for ESM dissemination (CMED) protocol has been developed based on the minimum delay multicast tree. To deal with the inherent nature of unreliability in wireless transmission, the tree-based multicast strategy is complemented and combined with contention-based relaying and implicit acknowledgment strategies. The CMED protocol consists of three main procedures: SendInitialMessage, SendMessage, and ReceiveMessage. Algorithm 2 describes the pseudocode of the CMED protocol.

An abnormal vehicle becomes a sender node which initiates the first ESM by invoking the SendInitialMessage procedure. In this procedure, a multicast tree is computed using Dijkstra's shortest path algorithm [16]. The input 


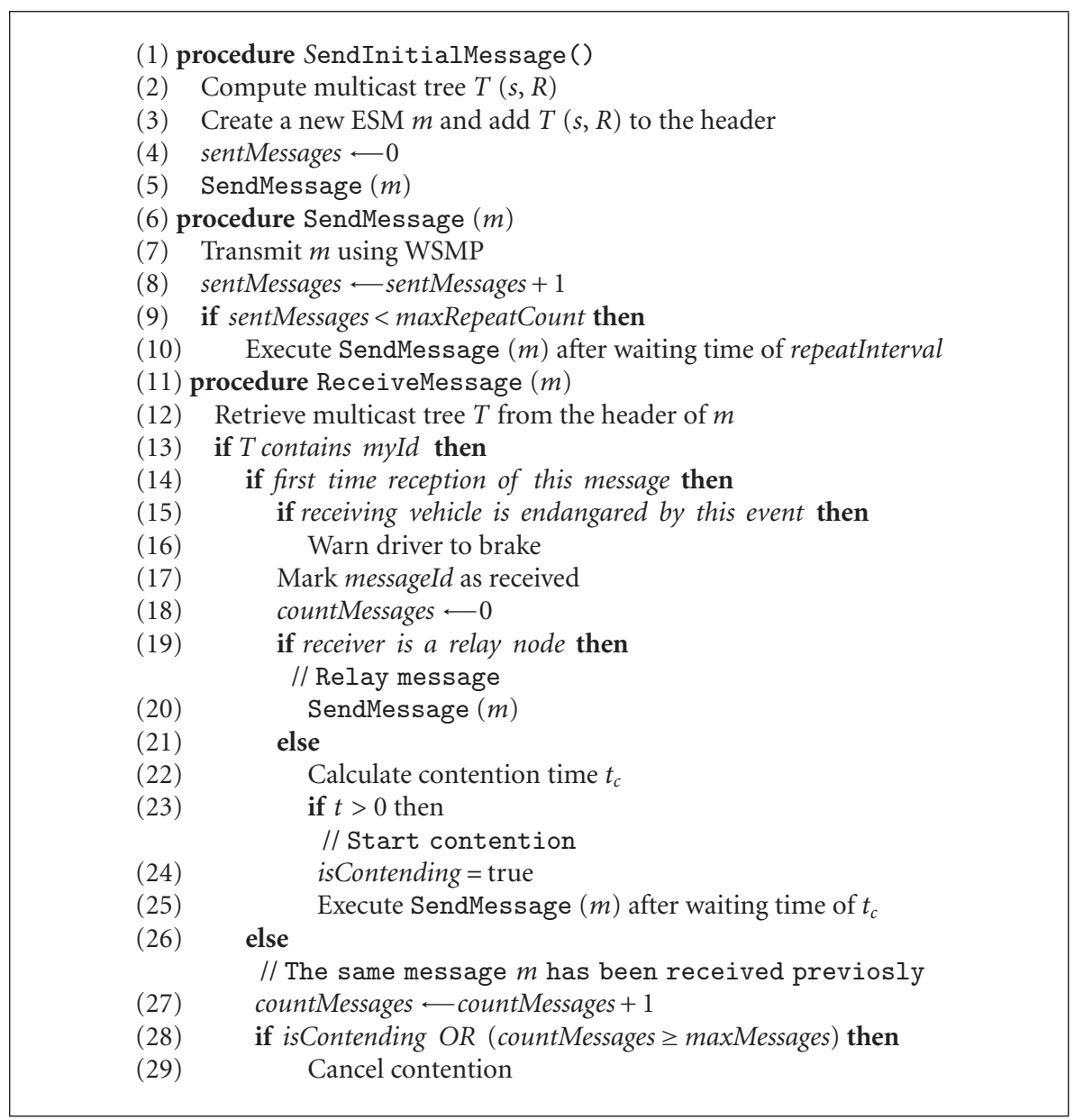

Algorithm 2: Pseudo-code of the CMED protocol.

values or parameters required by Dijkstra's algorithm are obtained from the context information

(1) The sender node $s$ is the abnormal vehicle that can be determined using methods described in Section 3.4.3.

(2) The network graph $G=(V, E)$ is generated using Algorithm 1.

(3) The receiver nodes are the endangered vehicles. The set of receiver nodes $R$ is determined using the vehicle interaction graph, as detailed in Section 3.4.4.

(4) The delay $\delta(v)$ for each node $v \in V$, that can be obtained using a method described in Section 3.4.2.

The output of the algorithm is the multicast tree $T(s, R)$, which is used as the routing paths to disseminate the ESMs. Next, a new ESM is created and the tree $T(s, R)$ is then encoded to the ESM. The ESM also contains an originId which is a unique identifier of the initial sender node (abnormal vehicle) and an eventId which is a unique identifier for each emergency event initiated by a node. The pair of originId and eventId represents a unique messageId which is used to identify a particular emergency event. After that, the SendMessage procedure is invoked to send the ESM.
The SendMessage procedure transmits a message using the WAVE Short Message Protocol (WSMP) as defined in the IEEE 1609.3 standard [40]. A node keeps track of the number of messages sent for each unique emergency event. To improve reliability, a sender will send the same message repeatedly with a constant interval of repeatInterval. The repetition is terminated if the same message is received from another node that belongs to the same multicast tree. In addition, a maximum number of repetitions is introduced to further reduce the channel load. The message will not be transmitted more than maxRepeatCount times.

The ReceiveMessage procedure is invoked when a node receives an ESM. The multicast tree is first retrieved from the message and the receiving node checks if the tree contains the node's unique identifier myId (such as a MAC address). The message will only be processed if the tree contains the receiver's id. If the same message has been received previously, it is then considered as an implicit acknowledgment, and any scheduled transmission for the same message will be canceled. If the message is received for the first time, the received messageId is recorded and the driver will be alerted. If the receiver node is a relay node, as identified from the multicast tree, the node will relay the message immediately. It is possible that a designated relay 
node may not receive the ESM or may not receive it in time. To ensure reliability, a contention-based method similar to other protocols [27-29] is used. Another receiver node may relay the message as a substitute to a failed relay node. If the receiver node is not a relay node, it will schedule to send the message after a specific contention time, which will be canceled if it receives another message with the same messageId. The contention time is calculated based on its distance to the sender node:

$$
t_{c}=\max \text { ContentionTime } \times\left(1-\frac{d_{\mathrm{sr}}}{d_{\max }}\right),
$$

where maxContention Time is a parameter of the maximum contention time, $d_{\mathrm{sr}}$ is the distance between the sender and the receiver, and $d_{\max }$ is the approximate transmission range.

\section{Performance Evaluation}

The performance of the CMED protocol was evaluated by simulation. The simulation was implemented in the network simulator ns-3 (version 3.8) [41]. The performance of the CMED protocol was compared with the following ESM protocols.

(1) Intelligent Broadcast with Implicit Acknowledgment (IBIA) protocol [12]: this protocol represents a simple approach to ESM dissemination in which the rebroadcasting strategy is based on random contention and vehicle direction.

(2) Emergency Message Dissemination for Vehicular environment (EMDV) protocol [28]: this protocol employs a distance-based contention strategy complemented with the selection of a next hop forwarder made at transmission time to minimize the delay.

All of the protocols were implemented as higher layer protocols that directly interface with the MAC layer protocol in ns-3.

4.1. Performance Metrics. The performance is measured in terms of the safety and communication issues. A protocol is better in terms of safety if using the protocol results in the smallest number of vehicle collisions. The communication issues involve the metrics of dissemination latency (or delay) and number of sent messages. The maximum dissemination latency is the time required to disseminate an ESM to all of the intended receivers. A lower dissemination time gives a better chance for a vehicle to avoid a collision. The number of sent messages reflects the efficiency of the protocol. A smaller number of sent messages indicates a more efficient protocol.

Vehicle collisions are mainly caused by vehicles that do not receive the ESM in time or do not receive the ESM at all. This means that safety performance is mostly influenced by the dissemination latency and the reliability of the communications. The reliability of a protocol is indirectly measured from the number of vehicle collisions. As a reference, the evaluation includes the results from a theoretical optimal protocol in which ESMs can be disseminated to all relevant vehicles with zero delay or latency.
In order to measure those metrics, a vehicle-following logic similar to the work proposed by Biswas et al. [12] has been developed. This was done by extending the ns-3 mobility model and developing a highway scenario module that can simulate road accidents, particularly a chain or multiple collisions on a highway. A chain collision situation is started by triggering an emergency event, in which a vehicle is forced to rapidly decelerate at $8 \mathrm{~m} / \mathrm{s}^{2}$. Such a vehicle becomes an abnormal vehicle. The high deceleration rate models an unexpected collision scenario in which a vehicle can stop within a short distance. Immediately after decelerating, the abnormal vehicle will start sending the ESMs. A vehicle that receives an ESM will start braking with a normal deceleration of $4.9 \mathrm{~m} / \mathrm{s}^{2}$ after a $1.5 \mathrm{~s}$ reaction time. A vehicle is not allowed to change lanes. Existing protocols for ESM dissemination were designed specifically for highway scenarios. Therefore, highway scenarios are chosen to allow a direct comparison with existing protocols.

4.2. Simulation Design and Setup. To provide a fair comparison, the simulation parameters and environment are configured to match the ones used by the authors of IBIA and EMDV $[12,28]$ as closely as possible. In the simulation, all vehicles are equipped with a CCWS. Each vehicle generates RSMs (beacon messages) at a rate of 10 messages per second. Before sending the first RSM, each vehicle calculates a random number from 0 to $100 \mathrm{~ms}$, and uses that number as the time to send the first RSM. The next RSM is then scheduled to be sent periodically every $100 \mathrm{~ms}$. The message size of both ESMs and RSMs is set to 500 bytes, including the application protocol specific headers. A constant message size is used to provide a consistent comparison result. The transmission power is configured to $19 \mathrm{dBm}$, which corresponds to $1000 \mathrm{~m}$ transmission range with around $20 \%$ message reception probability and $500 \mathrm{~m}$ transmission range with $90 \%$ message reception probability. The probabilistic Nakagami distribution is selected as the radio propagation loss model [28]. Real-world tests on highways showed that the Nakagami distribution is suitable to model the radio propagation in highway scenarios. The parameter of $m=$ 1 is set to simulate severe fading conditions, therefore, demonstrating the protocols' performance in the worst case scenario.

The lower layer protocol (PHY and MAC) parameters are set according to the IEEE 802.11p standard [1], which operates at $5.9 \mathrm{GHz}$ on a $10 \mathrm{MHz}$ control channel $(\mathrm{CCH})$. The PHY data rate is configured to $6 \mathrm{Mbps}$, which is the optimal value for safety communication [42]. The channel switching scheme is currently not implemented, which means the whole $10 \mathrm{MHz} \mathrm{CCH}$ bandwidth can be used by the CCWS application. The MAC layer is configured to ad hoc mode with QoS support using the EDCA mechanism as described in IEEE 802.11e. The priority for ESMs is set to $\mathrm{AC}_{-} \mathrm{VO}$ (highest), and the priority for RSMs is set to AC_VI (second highest). Both the ESM and RSM protocols are simulated as extensions to the IEEE 1609 WAVE Short Message Protocol (WSMP) [40]. They are directly implemented on top of the MAC layer. 
TABLE 1: Common configuration parameters.

\begin{tabular}{lc}
\hline Parameter & Value \\
\hline PHY and MAC protocol & $802.11 \mathrm{p}$ \\
$802.11 \mathrm{p}$ data rate & $6 \mathrm{Mbps}$ \\
Propagation loss model 1 & Three-log distance \\
Propagation loss model 2 & Nakagami $m=1$ \\
Transmission power & $19 \mathrm{dBm}$ \\
Safety message size & 500 bytes \\
RSM generation rate & 10 packets/s \\
Priority level: & \\
ESM & AC_VO \\
RSM & AC_VI \\
\end{tabular}

TABLE 2: Specific protocol parameters.

\begin{tabular}{lc}
\hline Parameter & Value \\
\hline IBIA messagePeriod & $100 \mathrm{~ms}$ \\
IBIA randomWaitTime & $(0-10) \mathrm{ms}$ \\
IBIA maxRepeatCount & 10 \\
EMDV disseminationAreaLength & $2000 \mathrm{~m}$ \\
EMDV forwardingRange & $500 \mathrm{~m}$ \\
EMDV maxMessages & 3 \\
EMDV maxContentionTime & $100 \mathrm{~ms}$ \\
EMDV maxChannelAccessTime & $10 \mathrm{~ms}$ \\
CMED maxContentionTime & $10 \mathrm{~ms}$ \\
CMED d max & $500 \mathrm{~m}$ \\
CMED maxRepeatCount & 10 \\
CMED repeatInterval & $100 \mathrm{~ms}$ \\
CMED maxMessages & 3 \\
\hline
\end{tabular}

The common configuration details related to lower layer protocols are summarized in Table 1 . The specific parameters to the IBIA and EMDV protocols are configured by following the given value in the original paper. A minor modification is made to the IBIA protocol by setting a maximum number of repetitions. The specific parameters for the IBIA, EMDV, and CMED protocols are detailed in Table 2.

The ESM protocol was evaluated in two typical cases of highway scenarios. The first case is a simple highway scenario that represents a typical road traffic scenario with relatively low communication traffic. The second case represents a typical road traffic scenario with high communication traffic density. In the second case, the distance between vehicles is randomized at each simulation run to test how the protocols perform in different highway traffic situations. Table 3 provides the common parameters that are used in all scenarios.

4.2.1. Simple Highway Scenario. This scenario models a single lane highway with $n$ vehicles moving in the same direction with the same speed, similar to the scenario used to evaluate the IBIA protocol [12]. The platoon was formed with uniform intervehicle spacing $s$, as illustrated in Figure 4(a). To demonstrate the protocols' performance with different vehicle densities, the simulation is conducted
TABLE 3: Common parameters for the highway scenario.

\begin{tabular}{lc}
\hline Parameter & Value \\
\hline Driver's reaction time & $1.5 \mathrm{~s}$ \\
Vehicle length & $4 \mathrm{~m}$ \\
Vehicle speed & $32 \mathrm{~m} / \mathrm{s}$ \\
Vehicle emergency deceleration & $8.0 \mathrm{~m} / \mathrm{s}^{2}$ \\
Vehicle normal deceleration & $4.9 \mathrm{~m} / \mathrm{s}^{2}$ \\
\hline
\end{tabular}

TABle 4: Parameters specific to the simple highway scenario.

\begin{tabular}{lc}
\hline Parameter & Value \\
\hline Highway length & $2000 \mathrm{~m}$ \\
Number of vehicles & $(40-200)$ \\
Number of abnormal vehicles & 1 \\
Number of lanes & 1 \\
Intervehicle spacing & $(10-50) \mathrm{m}$ \\
\hline
\end{tabular}

TABle 5: Parameters specific to the random highway scenario.

\begin{tabular}{lc}
\hline Parameter & Value \\
\hline Number of vehicles & 600 \\
Number of abnormal vehicles & $(1-6)$ \\
Number of lanes & 6 \\
Inter-vehicle spacing & $(9-50) \mathrm{m}$ \\
\hline
\end{tabular}

with different numbers of vehicles $n$ ranging from 40 to 200 . The distance between the vehicles at the first $\left(v_{0}\right)$ and last $\left(v_{n}\right)$ positions is fixed to 2000 meters, so a higher number of vehicles would mean a shorter intervehicle spacing. An emergency event was initiated by the abnormal vehicle at the front of the platoon $\left(v_{0}\right)$. Without a CCWS, all of the vehicles behind vehicle $v_{0}$ would be involved in chain collisions. This scenario is useful to evaluate the performance of ESM protocols in a low-density environment with deterministic node topology and mobility. Each simulation was repeated 100 times with a random seed for each run to obtain a statistically significant result. Table 4 indicates the specific parameters used in this scenario.

4.2.2. Random Highway Scenario. This scenario models a multilane bidirectional highway environment. Figure 4(b) illustrates this scenario. The highway consists of 6 lanes with 3 lanes for each direction. The total number of vehicles is 600 , and each lane contains 100 vehicles. The starting distance between vehicles was randomized for every simulation run, with a value between 9 and $50 \mathrm{~m}$. $N$ number of abnormal vehicles (initial sender) were randomly selected, which means $N$ emergency events were initiated in the course of the simulation. Those events were triggered consecutively with a $1 \mathrm{~ms}$ interval. Experiments are conducted for different values of $N$ ranging from 1 to 6 . Each time, the simulation is repeated with a random seed 100 times to obtain a statistically significant result. Table 5 indicates the specific parameters used in this scenario. 


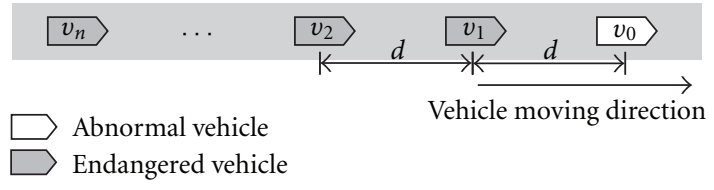

(a) Simple highway scenario

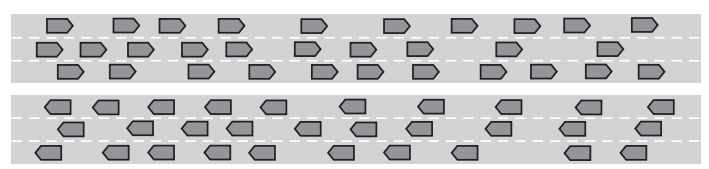

(b) Random highway scenario

FIGURE 4: Illustration of highway scenarios used in the simulation.

\subsection{Simulation Results}

4.3.1. Simple Highway Scenario. From 100 simulation runs, the average of maximum dissemination latency for scenarios of 40 to 200 vehicles is calculated and plotted in Figure 5. The result shows that a higher number of vehicles (higher density) leads to a higher latency. In all of the cases, the CMED protocol can deliver ESMs faster than the IBIA and EMDV protocols. In terms of efficiency, the number of sent messages is plotted in Figure 6. It shows that the number of sent messages for the CMED protocol is just slightly lower than for the EMDV protocol. Both the CMED and EMDV protocols send significantly fewer messages compared to the IBIA protocol. In this scenario, all of the protocols can achieve an optimal number of vehicle collisions in most cases. Table 6 shows that there is no significant difference in the numbers of collisions resulting from this scenario between the three protocols. For example, in a case with 200 vehicles, the total number of collisions averaged from 100 simulation instances was 15.97 for IBIA, 14.14 for EMDV, and 14.11 for CMED. As a reference, the smallest number of collisions that can be achieved is 14 . The higher number of collisions for IBIA means that in some instances of the simulation, the protocol has failed to ensure 100\% message delivery to all endangered vehicles.

4.3.2. Random Highway Scenario. Each simulation instance produces a unique highway scenario that results in different number of vehicle collisions for each run. The lowest possible number of collisions for each unique scenario, which is the optimal result, is obtained by simulating a dummy protocol that can transmit ESMs to all relevant vehicles instantaneously just after an emergency event occurred. The resulting number of vehicle collisions for the other protocols was normalized to the optimal result in order to clearly show the differences between the evaluated protocols.

Figure 7 shows the number of excess vehicle collisions for different numbers of concurrent events (from 1 to 6). When there are more than one concurrent events, the CMED protocol delivers a lower number of collisions compared to both the IBIA and EMDV protocols. Given only one concurrent event, the CMED protocol delivers an equal

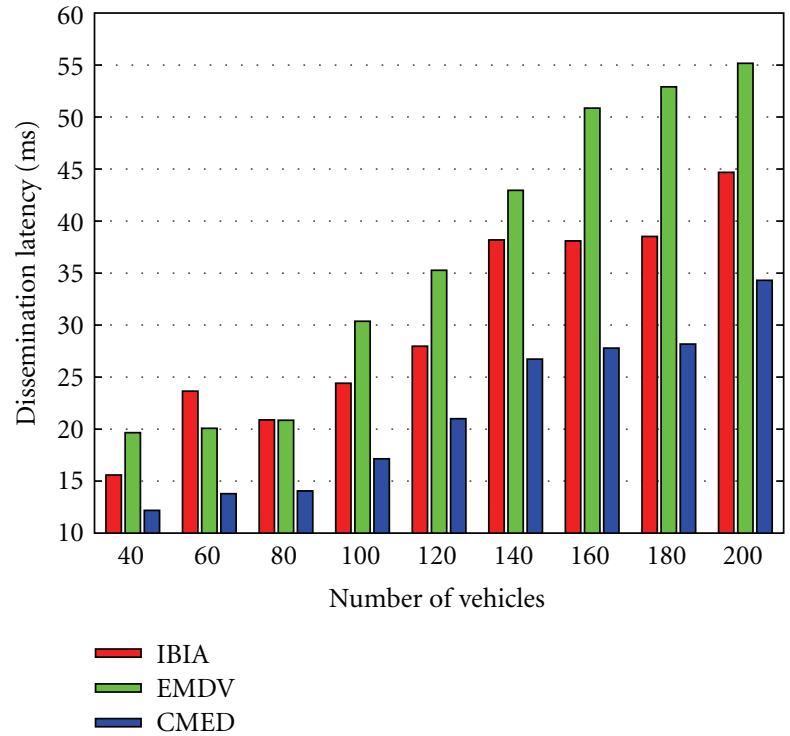

FIGURE 5: Average of maximum dissemination latency in the simple highway scenario.

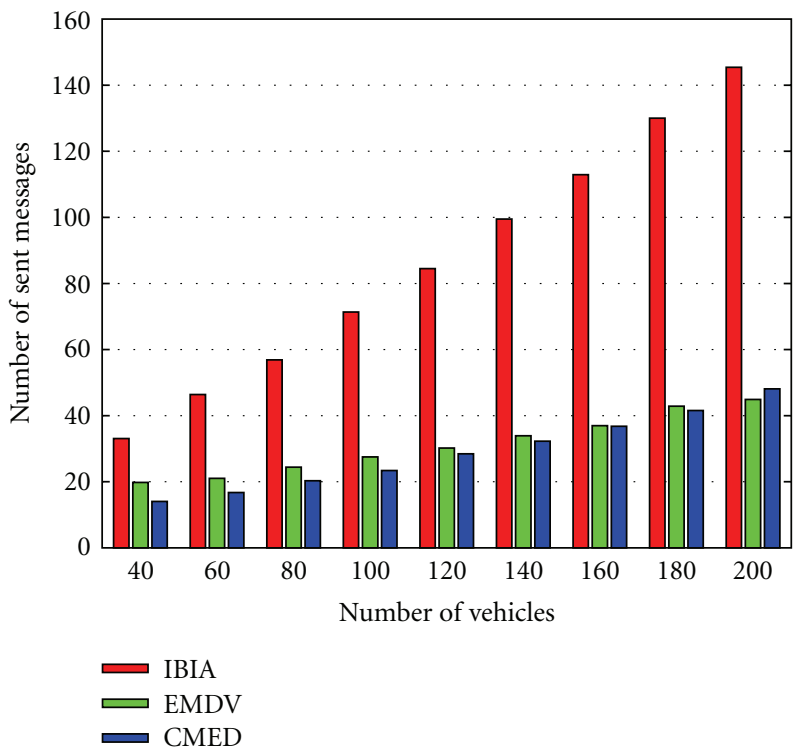

Figure 6: Total number of ESMs sent by all vehicles in the simple highway scenario.

number of collisions to the EMDV protocol, but lower than for the IBIA protocol.

The number of sent messages for the CMED protocol is the lowest for all of the cases, as shown in Figure 8. The overall number was obtained by adding up the number of messages sent by all vehicles from 100 simulation instances.

Figure 9 shows the maximum dissemination time required for an ESM to reach all relevant vehicles. The maximum dissemination time was averaged from 100 simulation instances. It shows that, generally, the CMED protocol is always able to disseminate the ESMs faster than either the IBIA and EMDV protocols. 
TABLE 6: Number of vehicle collisions in the simple highway scenario.

\begin{tabular}{lccccccccc}
\hline Number of vehicles & 40 & 60 & 80 & 100 & 120 & 140 & 160 & 180 & 200 \\
\hline IBIA & 1.2 & 3.24 & 4.03 & 5.38 & 6.7 & 9.25 & 11.52 & 13.49 & 15.97 \\
EMDV & 1.03 & 3 & 4 & 5 & 6.21 & 8 & 10.01 & 12.01 & 14.14 \\
CMED & 1 & 3 & 4 & 5 & 6.21 & 8.01 & 10 & 12.01 & 14.11 \\
\hline
\end{tabular}

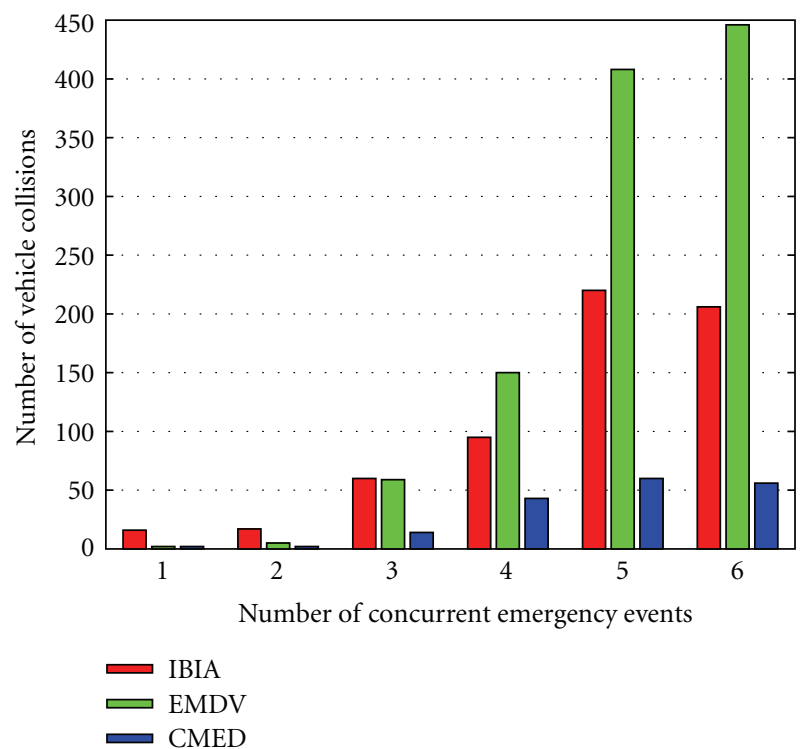

Figure 7: Number of excess vehicle collisions (normalized to the optimal results) from 100 instances of the random highway scenario.

\section{Discussion}

The simulation results demonstrate the advantages of the proposed CMED protocol. In the simple highway scenarios, the IBIA protocol results in lower latency, but a higher number of vehicle collisions, compared to the EMDV protocol. In some simulation instances, the IBIA protocol is not able to deliver warning messages to some endangered vehicles, which means that the IBIA protocol is less reliable compared to the EMDV and CMED protocols. Although the number of vehicle collisions for the CMED protocol is not much different compared to the EMDV protocol, the CMED protocol still shows some advantages in terms of the number of sent message and the maximum dissemination time.

The random highway scenarios demonstrate the performance of the protocols in a typical highway environment with high communication traffic density. Using the CMED protocol results in the fewest number of vehicle collisions. The results show that the CMED protocol is more reliable and scalable than the other protocols. It can generally improve the safety performance of the EEBL application. Figure 7 shows that for IBIA and EMDV protocols, the difference in the number of vehicle collisions becomes much larger as the number of concurrent events increases. A higher number of concurrent events means higher communication traffic density.

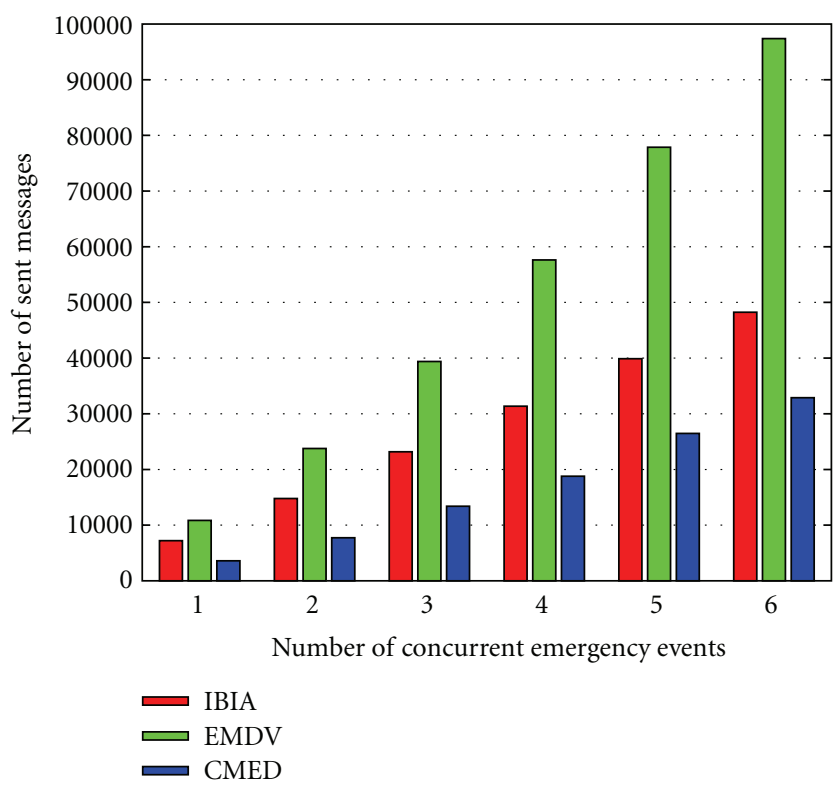

FIGURE 8: Total number of ESMs sent by all vehicles from 100 instances of the random highway scenario.

The number of sent messages shown in Figure 8 demonstrates that the CMED protocol is more efficient compared to both the IBIA and EMDV protocols. The CMED protocol is able to reduce the number of redundant transmissions without compromising safety by sending ESMs only to the relevant vehicles. Depending on the traffic conditions, ESMs may be disseminated only to some vehicles behind an abnormal vehicle. In contrast, the other protocols always disseminate ESMs to all vehicles behind an abnormal vehicle until there is no other vehicle in the radio range of the last relay.

The number of sent messages directly correlates with the channel usage level. In the 802.11-based wireless networks, higher channel usage will result in frequent backoffs and higher chance of packet collisions, which will degrade network performance and congest the channel as its capacity is reached [10]. The effect of network performance to safety is measured by the number of vehicle collisions. The simulation results show that the number of excess vehicle collisions increases as the number of sent messages increases. By using the channel efficiently, the CMED protocol is able to significantly improve overall network performance, particularly in the scenarios that can potentially create a very high channel usage.

The CMED protocol can achieve the lowest number of vehicle collisions because the protocol can disseminate ESMs with a low latency and a low rate of packet loss. The efficiency 


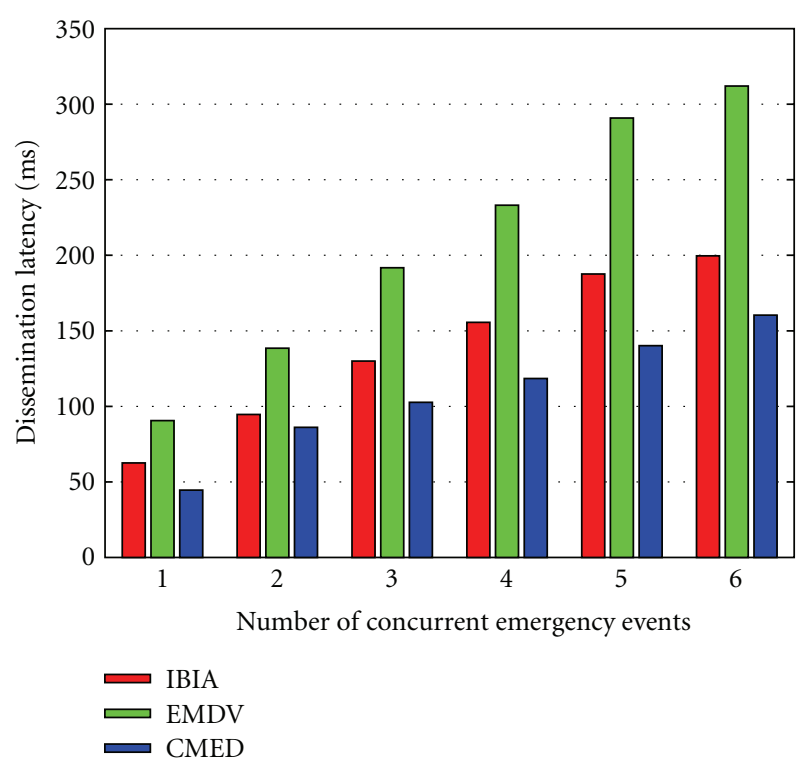

Figure 9: Maximum dissemination latency averaged from 100 instances of the random highway scenario.

of the protocol is one of the factors that contributes to a low latency and a low rate of packet loss. Another factor that improves the latency is the use of routing paths that is based on the least-delay multicast tree. Furthermore, the contention-based method of the CMED protocol addresses the possibility of packet loss and is able to improve the successful packet reception rate.

The use of context information in CMED protocol requires a larger computational overhead compared to other protocols. Since ns-3 is a discreet event-based simulator, it is not possible to measure the computational cost of any protocol and to integrate the measurement into the network simulation. Therefore, we have conducted a separate analysis and evaluation of the computational complexity of the CMED protocol. The main costly algorithms are the interaction graph and the multicast tree generations. As the interaction graph is updated regularly every time an RSM is received, its overhead does not directly affect the communication delay and each update is very fast because it has a complexity of $O(\mathcal{V})$. The multicast tree is generated by running the Dijkstra's algorithm, that has a complexity of $O\left(|V|^{2}\right)$. We have developed a Java-based tool to evaluate and visualize the interaction graph, and performed an experiment using the tool in a personal computer with a $2.5 \mathrm{GHz}$ processor. Given 600 vehicles, each $O(\mathcal{V})$ iteration of interaction graph generation only takes about less than 0.1 milliseconds to process. Our simple implementation of Dijkstra's algorithm can generate a multicast tree to 100 random receivers within less than 10 milliseconds. Using a more advance implementation can further reduce the complexity of Dijkstra's algorithm to $O(\log |V|)$. Even with 10 milliseconds extra latency, the overall CMED latency in the random scenario is still the lowest one. Based on the analysis and evaluation results, the extra computational

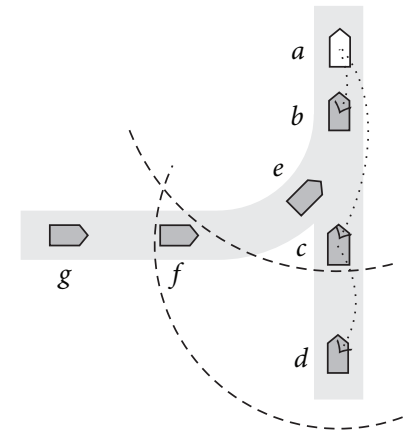

Abnormal vehicle

Endangered vehicle

(a)

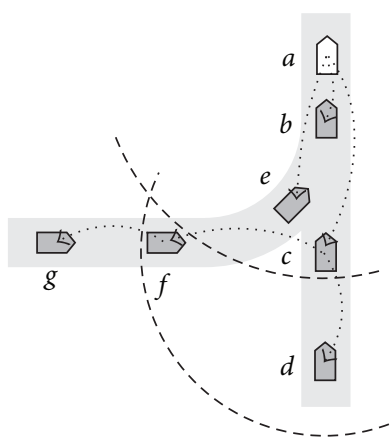

Abnormal vehicle

Endangered vehicle

(b)
Figure 10: Comparison of ESM dissemination in a junction road traffic scenario. (a) Using prior approaches. (b) Using the contextaware multicast approach.

cost does not significantly affect the simulation result of CMED.

In addition to the advantages in a highway scenario shown by the simulation results, another advantage of the CMED protocol is the applicability in various cases of traffic accidents. Existing ESM protocols only consider a straight road scenario such as a highway and assume that the receivers are those vehicles located within the region behind or in front of the abnormal vehicle that are moving towards the abnormal vehicle. However, some accidents may occur in a more complex road traffic situation such as at a junction, on a curved road, or a roundabout. Another weakness of prior ESM protocols is that for each transmission, only one relay can be selected based on the contention method, resulting in a single dissemination direction.

There are possibilities and variations of road traffic scenarios where ESMs must be disseminated in more than one direction. Figure 10 shows one example of a junction road topology where the endangered vehicles or broadcast region cannot be determined based on the forward or backward direction of the sender. In this example, assume that vehicle $a$ is the abnormal vehicle and vehicles $b, c, d, e$, $f$, and $g$ are the endangered vehicles. Figure 10(a) shows an example of ESM dissemination using the prior approaches. Vehicle $a$ broadcasts the ESM, which is received by vehicles $b, c$, and $e$. Vehicle $e$ ignores the received ESM because of its different direction. Vehicle $c$ is chosen as the relay based on the contention rules (e.g., farthest distance from the sender) and forwards the ESM to vehicle $d$. Vehicle $f$ ignores the received ESM for the same reason as vehicle $e$. This results in endangered vehicles $e, f$, and $g$ not receiving the ESM.

The CMED protocol uses a context-aware strategy to decouple the task of determining the endangered vehicles from the routing algorithm. The interaction graph, which is used to identify the endangered vehicles, can be considered as a separate module from the routing algorithm. Given 
the set of endangered vehicles as the input parameter, the routing algorithm can find the best possible paths to all of the endangered vehicles. Figure 10(b) shows the example of ESM dissemination using the CMED approach. Using this approach, vehicles $e$ and $f$ can recognize themselves as endangered vehicles, and vehicle $f$ is informed to forward the ESM to vehicle $g$. A protocol designed with this approach will be able to deliver the warning messages effectively in various road traffic scenarios.

\section{Conclusion and Future Work}

In this paper, we have presented a context-aware multicast protocol for ESM dissemination that can reduce the number of accidents or crashed vehicles. Differing from other existing approaches, the proposed multicast approach uses a precomputed routing tree based on the estimated delay and utilizes the vehicle interaction graph to identify receiver nodes. The multicast tree is the minimum delay tree from a sender to the receivers, computed using Dijkstra's shortest path algorithm with transmission delay as the cost function. The approach improves the wireless channel usage by reducing the number of sent messages and improves the dissemination time by using the least-delay path. Furthermore, the multicast protocol incorporates the contention-based forwarding techniques to improve reliability.

The performance of the proposed protocol has been evaluated by experiments conducted using the network simulator ns-3. It has been compared with broadcast protocols in highway traffic scenarios, with a constant beaconing load. Road accident scenarios are simulated by extending ns-3 with a new highway mobility model. The simulation results have shown that the multicast protocol outperforms the existing broadcast protocols in terms of efficiency and reliability. Moreover, the multicast protocol is able to significantly reduce accident casualties in some scenarios. In addition, the multicast approach can disseminate ESMs in any direction depending on the multicast tree, and therefore can support various road traffic scenarios.

Currently, the proposed multicast scheme requires context information about neighboring vehicles within more than 1-hop communication range. In future work, we will investigate a different approach that only needs 1hop information by extending and modifying the proposed multicast scheme. In addition, more experiments are needed to evaluate how the multicast protocol performs on other complex scenarios such as intersection and junction.

\section{Acknowledgments}

This work is supported by Queensland University of Technology and the Commonwealth of Australia, through the Cooperative Research Centre for Advanced Automotive Technology (AutoCRC) under Project C3-10. Computational resources and services used in this work are provided by the High Performance Computing and Research Support Unit, Queensland University of Technology, Brisbane, Australia.

\section{References}

[1] IEEE Std 802.11p, "IEEE Standard for Information technology_Local and metropolitan area networks-Specific requirements-Part 11: Wireless LAN Medium Access Control (MAC) and Physical Layer (PHY) Specifications Amendment 6: Wireless Access in Vehicular Environments," 2010.

[2] D. Jiang and L. Delgrossi, "IEEE 802.11p: towards an international standard for wireless access in vehicular environments," in Proceedings of the IEEE 67th Vehicular Technology Conference-Spring (VTC'08), pp. 2036-2040, May 2008.

[3] R. Miller and Q. Huang, "An adaptive peer-to-peer collision warning system," in Proceedings of the 55th Vehicular Technology Conference, pp. 317-321, May 2002.

[4] H. S. Tan and J. Huang, "DGPS-based vehicle-to-vehicle cooperative collision warning: Engineering feasibility viewpoints," IEEE Transactions on Intelligent Transportation Systems, vol. 7, no. 4, pp. 415-427, 2006.

[5] R. Sengupta, S. Rezaei, S. E. Shladover, D. Cody, S. Dickey, and H. Krishnan, "Cooperative collision warning systems: concept definition and experimental implementation," Journal of Intelligent Transportation Systems: Technology, Planning, and Operations, vol. 11, no. 3, pp. 143-155, 2007.

[6] G. K. Mitropoulos, I. S. Karanasiou, A. Hinsberger et al., "Wireless local danger warning: cooperative foresighted driving using intervehicle communication," IEEE Transactions on Intelligent Transportation Systems, vol. 11, no. 3, pp. 539-553, 2010.

[7] M. Torrent-Moreno, D. Jiang, and H. Hartenstein, "Broadcast reception rates and effects of priority access in 802.11-based vehicular ad-hoc networks," in Proceedings of the 1st ACM International Workshop on Vehicular Ad Hoc Networks, pp. 1018, ACM, Philadelphia, Pa, USA, October 2004.

[8] J. Yin, T. Elbatt, G. Yeung et al., "Performance evaluation of safety applications over DSRC vehicular ad hoc networks," in Proceedings of the 1st ACM International Workshop on Vehicular Ad Hoc Networks, pp. 1-9, ACM, Philadelphia, Pa, USA, October 2004.

[9] S. Eichler, "Performance evaluation of the IEEE $802.11 \mathrm{p}$ WAVE communication standard," in Proceedings of the 66th IEEE Vehicular Technology Conference (VTC '07), pp. 21992203, October 2007.

[10] Y. Zang, L. Stibor, X. Cheng, H.-J. Reumerman, A. Paruzel, and A. Barroso, "Congestion control in wireless networks for vehicular safety applications," in Proceedings of the 13th European Wireless Conference, Paris, France, April 2007.

[11] D. Jiang, V. Taliwal, A. Meier, W. Holfelder, and R. Herrtwich, "Design of 5.9 GHz DSRC-based vehicular safety communication," IEEE Wireless Communications, vol. 13, no. 5, pp. 36-43, 2006.

[12] S. Biswas, R. Tatchikou, and F. Dion, "Vehicle-to-vehicle wireless communication protocols for enhancing highway traffic safety," IEEE Communications Magazine, vol. 44, no. 1, pp. 74-82, 2006.

[13] "The CAMP Vehicle Safety Communications Consortium. Vehicle safety communications project-task 3 final reportidentify intelligent vehicle safety applications enabled by DSRC," Technical Report DOT HS 809 859, National Highway Traffic Safety Administration, US Department of Transportation, Washington, DC, USA, 2005.

[14] The Local, "Dozens injured in 259-car autobahn pileup," 2009, http://www.thelocal.de/national/20090720-20701.html. 
[15] P. Alexander, D. Haley, and A. Grant, "Cooperative intelligent transport systems: 5.9-GHz field trials," Proceedings of the IEEE, vol. 99, no. 7, pp. 1213-1235, 2011.

[16] E. W. Dijkstra, "A note on two problems in connexion with graphs," Numerische Mathematik, vol. 1, no. 1, pp. 269-271, 1959.

[17] H. Yao Ho, H. Ai Ho, and A. Kien Hua, "Routing protocols for intervehicular networks: a comparative study in highmobility and large obstacles environments," Computer Communications, vol. 31, no. 12, pp. 2767-2780, 2008.

[18] S.-Y. Ni, Y.-C. Tseng, Y.-S. Chen, and J.-P. Sheu, "The broadcast storm problem in a mobile ad hoc network," in Proceedings of the 5th ACM/IEEE International Conference on Mobile Computing and Networking, pp. 151-162, ACM, Seattle, Wash, USA, 1999.

[19] X. Yang, J. Liu, F. Zhao, and N. H. Vaidya, "A vehicleto-vehicle communication protocol for cooperative collision warning," in Proceedings of 1st Annual International Conference on Mobile and Ubiquitous Systems: Networking and Services (MOBIQUITOUS '04), pp. 114-123, Boston, Mass, USA, August 2004.

[20] A. Benslimane, "Optimized dissemination of alarm messages in Vehicular Ad-Hoc Networks (VANET)," in High Speed Networks and Multimedia Communications, vol. 3079 of Lecture Notes in Computer Science, pp. 655-666, Springer, Berlin, Germany, 2004.

[21] E. Fasolo, A. Zanella, and M. Zorzi, "An effective broadcast scheme for alert message propagation in vehicular ad hoc networks," in Proceedings of the IEEE International Conference on Communications (ICC '06), vol. 9, pp. 3960-3965, July 2006.

[22] A. Amoroso, G. Marfia, and M. Roccetti, "Going realistic and optimal: a distributed multi-hop broadcast algorithm for vehicular safety," Computer Networks, vol. 55, no. 10, pp. 2504 2519, 2011.

[23] C. E. Palazzi, S. Ferretti, M. Roccetti, G. Pau, and M. Gerla, "How do you quickly choreograph inter-vehicular communications? A fast vehicle-to-vehicle multi-hop broadcast algorithm, explained," in Proceedings of the 4th Annual IEEE Consumer Communications and Networking Conference (CCNC'07), pp. 960-964, January 2007.

[24] N. Wisitpongphan, O. K. Tonguz, J. S. Parikh, P. Mudalige, F. Bai, and V. Sadekar, "Broadcast storm mitigation techniques in vehicular ad hoc networks," IEEE Wireless Communications, vol. 14, no. 6, pp. 84-94, 2007.

[25] R. S. Schwartz, R. R. R. Barbosa, N. Meratnia, G. Heijenk, and H. Scholten, "A directional data dissemination protocol for vehicular environments," Computer Communications, vol. 34, no. 17, pp. 2057-2071, 2011.

[26] M. N. Mariyasagayam, T. Osafune, and M. Lenardi, "Enhanced Multi-hop Vehicular Broadcast (MHVB) for active safety applications," in Proceedings of the 7th International Conference on Intelligent Transport Systems Telecommunications (ITST '07), pp. 223-228, June 2007.

[27] Y. T. Yang and L. D. Chou, "Position-based adaptive broadcast for inter-vehicle communications," in Proceedings of the IEEE International Conference on Communications Workshops (ICC '08), pp. 410-414, May 2008.

[28] M. Torrent-Moreno, J. Mittag, P. Santi, and H. Hartenstein, "Vehicle-to-vehicle communication: fair transmit power control for safety-critical information," IEEE Transactions on Vehicular Technology, vol. 58, no. 7, pp. 3684-3703, 2009.

[29] S. Yu, M. Lee, and G. Cho, "An emergency message propagation method in highway traffic," in Proceedings of the
Ubiquitous Computing Systems (UCS '06), Lecture Notes in Computer Science, pp. 331-343, Springer, Berlin, Germany, 2006.

[30] M. M. I. Taha and Y. M. Y. Hasan, "VANET-DSRC protocol for reliable broadcasting of life safety messages," in Proceedings of the IEEE International Symposium on Signal Processing and Information Technology (ISSPIT '07), pp. 104-109, December 2007.

[31] A. Sebastian, M. Tang, Y. Feng, and M. Looi, "A multicast routing scheme for efficient safety message dissemination in VANET," in Proceedings of the IEEE Wireless Communications and Networking Conference (WCNC '10), Sydney, Australia, April 2010.

[32] V. Kukshya, H. Krishnan, and C. Kellum, "Performance evaluation of a system for estimating relative positions of vehicles during GPS outages," in Proceedings of the IEEE Intelligent Vehicles Symposium (IV '06), pp. 394-399, ACM, Tokyo, Japan, June 2006.

[33] R. Parker and S. Valaee, "Vehicular node localization using received-signal-strength indicator," IEEE Transactions on Vehicular Technology, vol. 56, no. 6, pp. 3371-3380, 2007.

[34] A. Boukerche, H. A. B. F. Oliveira, E. F. Nakamura, and A. A. F. Loureiro, "Vehicular Ad Hoc networks: a new challenge for localization-based systems," Computer Communications, vol. 31, no. 12, pp. 2838-2849, 2008.

[35] N. M. Drawil and O. Basir, "Intervehicle-communicationassisted localization," IEEE Transactions on Intelligent Transportation Systems, vol. 11, no. 3, pp. 678-691, 2010.

[36] J. Mittag, F. Thomas, J. Härri, and H. Hartenstein, "A comparison of single- and multi-hop beaconing in VANETs," in Proceedings of the 6th ACM International Workshop on VehiculAr Inter-NETworking (VANET '09), pp. 69-78, ACM, New York, NY, USA, September 2009.

[37] K. Ibrahim and M. C. Weigle, "Accurate data aggregation for VANETs," in Proceedings of the 4th ACM International Workshop on Vehicular Ad Hoc Networks (VANET'07), pp. 7172, ACM, Quebec, Canada, September 2007.

[38] A. Munaretto and M. Fonseca, "Routing and quality of service support for mobile ad hoc networks," Computer Networks, vol. 51, no. 11, pp. 3142-3156, 2007.

[39] A. Sebastian, M. Tang, Y. Feng, and M. Looi, "Multivehicles interaction graph model for cooperative collision warning system," in Proceedings of the IEEE Intelligent Vehicles Symposium, pp. 929-934, Xi'an, China, June 2009.

[40] IEEE Std 1609.3, "IEEE Standard for Wireless Access in Vehicular Environments (WAVE)—Networking Services," pp. $1-144,2010$.

[41] “The ns-3 network simulator," 2012, http://www.nsnam.org/.

[42] D. Jiang, Q. Chen, and L. Delgrossi, "Optimal data rate selection for vehicle safety communications," in Proceedings of the 5th ACM International Workshop on VehiculAr InterNETworking (VANET '08), pp. 30-38, ACM, San Francisco, Calif, USA, September 2008. 

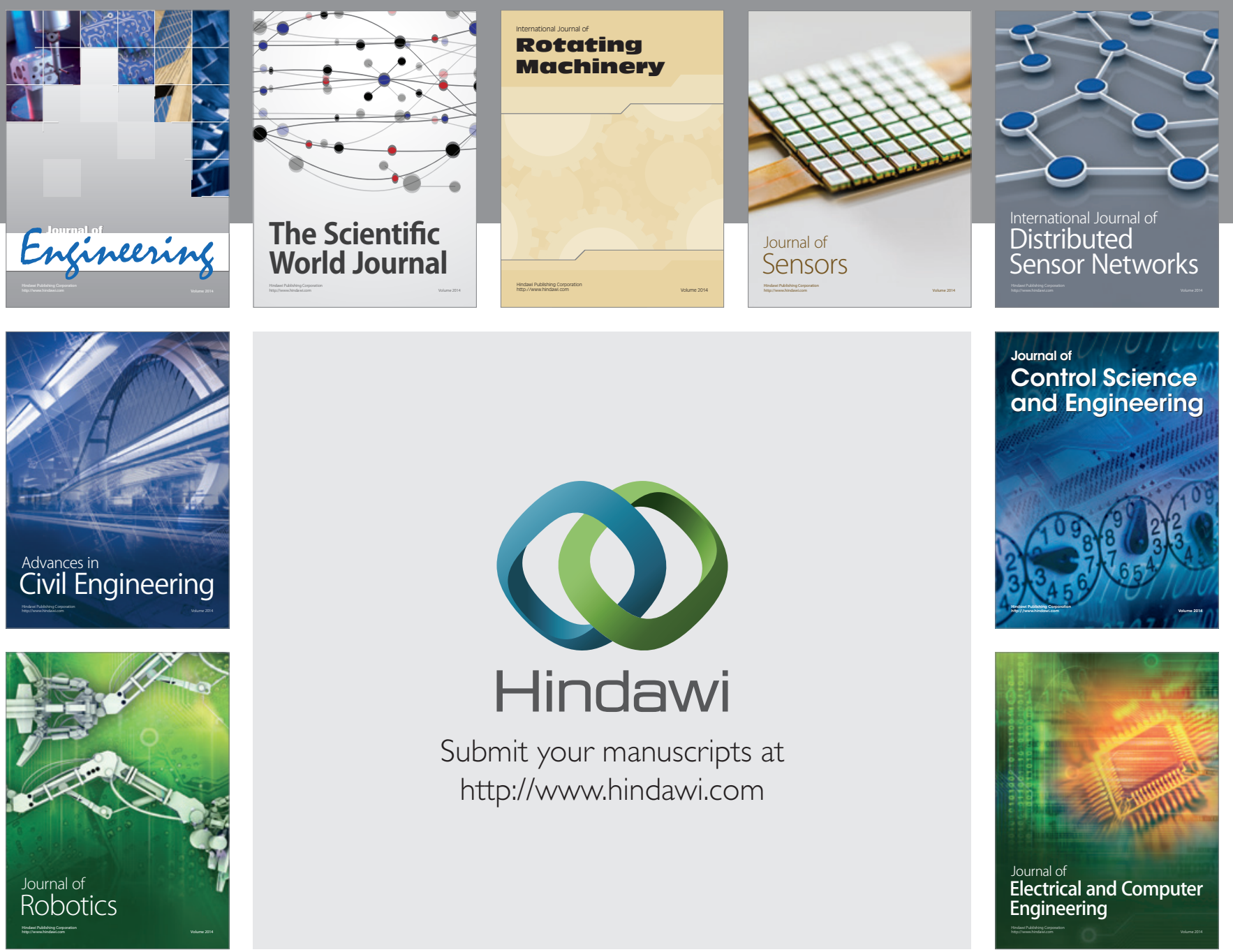

Submit your manuscripts at

http://www.hindawi.com
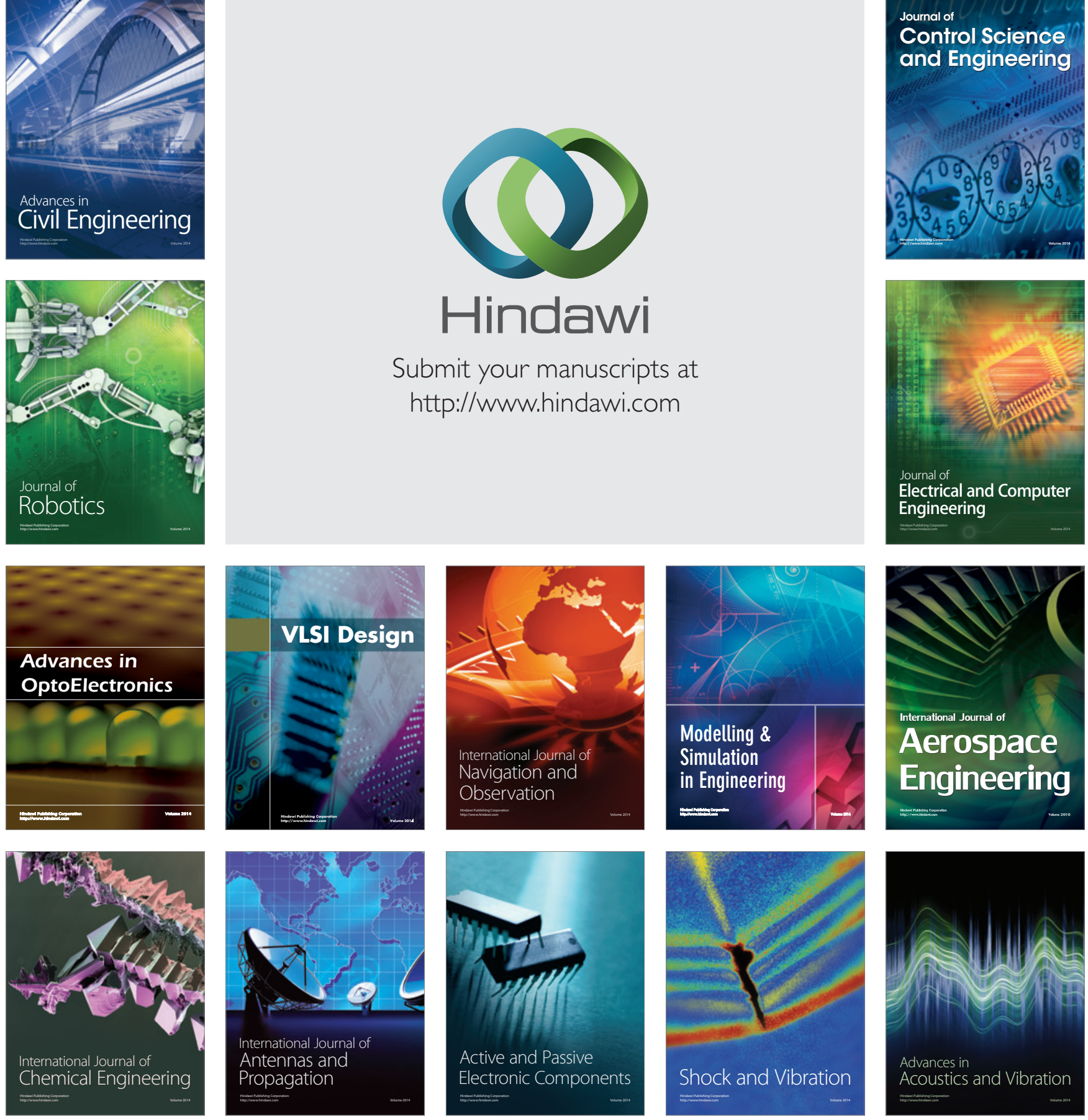